\title{
Empirical Neutron Cross Section Formulae for $(n, p)$ and (n, d) Reactions of He-3 Target
}

\author{
Amir M. Alramady \\ King Abdulaziz University, Jeddah, KSA \\ Email: ameerkamel@hotmail.com,Amelramady@kau.edu.sa
}

How to cite this paper: Alramady, A.M. (2021) Empirical Neutron Cross Section Formulae for (n, p) and (n, d) Reactions of He-3 Target. Journal of Applied Mathematics and Physics, 9, 1434-1441. https://doi.org/10.4236/jamp.2021.97096

Received: June 5, 2021

Accepted: July 2, 2021

Published: July 5, 2021

Copyright (อ 2021 by author(s) and Scientific Research Publishing Inc. This work is licensed under the Creative Commons Attribution International License (CC BY 4.0).

http://creativecommons.org/licenses/by/4.0/ (c) (i) Open Access

\begin{abstract}
In this work, new cross section formulae for (n, p) and (n, d) reactions of He-3 have been investigated as a function of incident neutron energy. The new Empirical formulae were produced by using the least squares method to the experimental cross sections data, which were taken from Experimental Nuclear Reaction Data EXFOR Database Version of 2021. Several functions were examined to choose the best one that fits the data. Statistical hypothesis testing was used to insure how well the suggested equations fit the set of data. Two statistical indicators were used for each case for goodness-of-fit. Very high compatibility was found between the empirical values and the experimental data for both reactions.
\end{abstract}

\section{Keywords}

Empirical Cross Section Formula, (n, p) Reaction, (n, p) Reaction, and Goodness-of-Fit

\section{Introduction}

The nucleus helium-3 (He-3) which proposed for the first time by the Mark Oliphant in 1934 [1] has very much importance in the field of radiation instrumentation and nuclear safeguards [2] and [3]. Neutron capture of the He-3 nucleus is an indication of a nuclear reaction in which a neutron (n) bombards $\mathrm{He}-3$ results in the emission of a proton (p), deuteron (d), tritium (T), or $\gamma$-ray. All these interactions are of special importance in a lot of scientific fields [4] and [5]. For example, He-3(n, p) H-3 reaction has particular importance in nuclear well-logging techniques in physical petroleum research [6].

Cross section data may be needed during calculations of nuclear systems, medical applications, nuclear industrial studies, or other nuclear fields. Some 
nuclear database libraries such as the Evaluated Nuclear Data File (ENDF), Japanese Evaluated Nuclear Data Library (JENDL), TALYS-based Evaluated Nuclear Data Library (TENDL), and Joint Evaluated Fission and Fusion File (JEFF) are available for $(n, d)$ reactions but theses libraries do not cover all the required ranges of cross sections [7]-[12]. For this purpose, a new cross-section formula for some neutron-deuteron reactions as a function of incident neutron energy has been created by current work.

"Curve Fitting" term indicates to the mathematical method that one can find the formula which can be represented as: $y=f(x)$ that can be the best fit with the corresponding $y$-values and the set of observed $x$-values [13].

For the mathematical method, the creation of the best-fit formulas for the observed set of points was to reduce the sum of squares of the residuals of the points from the curve. This method is called the least square method [14].

By assuming that the observed data are in the form of Cartesian pairs $\left(x_{i}, y_{i}\right)$, $1 \leq i \leq N$, and the suggested empirical function $Y\left(X_{i}\right)$ is assumed to be a straight line with the equation:

$$
Y\left(X_{i}\right)=a * X_{i}+b
$$

The $i^{\text {th }}$ error $\operatorname{Er}(i)$ of the estimation data is then equaling to:

$$
\operatorname{Er}(i)=Y\left(X_{i}\right)-a-b X_{i}
$$

Then, total squared error " $E$ " can be defined as:

$$
E=\sum_{i=1}^{N}\left(y_{i}-a-b X_{i}\right)^{2}
$$

where $N$ is the number of the observed pairs of data and $E$ s subscript refers to the solution's order.

The lower the value of $E$, the closer the proposed solution is to the observed data. For calculation of $a$ and $b$ constants, $E$ must be very small. By partial derivation of Equation (3) with respect to $a$ and $b$ two times and making them equal to zero, the constants $a$ and $b$ can be deduced.

$$
\begin{gathered}
\frac{\partial}{\partial a} E=\frac{\partial}{\partial b} E=0 \\
\frac{\partial}{\partial a}(E)=\frac{\partial}{\partial a} \sum_{i=1}^{N}\left(y_{i}-a-b X_{i}\right)^{2}=0 \\
\frac{\partial 2}{\partial b}(E)=-2 * \sum_{i=1}^{N} y_{i}-a-b X_{i}=0
\end{gathered}
$$

So

$$
\begin{gathered}
\sum_{i=1}^{N} y_{i}=a * N+b * \sum_{i=1}^{N} \operatorname{Er}(i) \\
\sum_{i=1}^{N} y_{i} * \operatorname{Er}(i)=a * \sum_{i=1}^{N} \operatorname{Er}(i)+b * \sum_{i=1}^{N} \operatorname{Er}(i)^{2}
\end{gathered}
$$

Parameters $a$ and $b$ are found from the solution of Equations (7) and (8). For 
general case, least-squares method is applied to reach the best solutions by finding the best possible values for a group of parameters.

Parametric fitting includes discovering parameters for at least one show that you fit to information. The information is thought to be measurable in nature and is separated into two segments: deterministic and arbitrary. The deterministic part is given by a parametric model and the arbitrary segment is frequently depicted as blunder related with the data. We can say that the observed data is equal to the sum of both parametric model and the error.

A goodness-of-fit test or indicator is performed to statistically examine whether the estimated formula gives a good description of the observed data. Nash-Sutcliffe efficiency indicator NSE and root mean square error indicator RMSE are two of the most popular indicators for goodness of fit [15] [16] and [17].

If " $N$ " is the number of observed points, then RMSE is defined as:

$$
\text { RMSE }=\frac{1}{N} \sum_{1}^{N}\left(y_{i}-Y_{i}\right)^{2}
$$

Its value ranges from zero to infinity, where zero value indicates the best fit.

The second goodness-of-fit indicator is the dimensionless indicator NSE and can be calculated as [15]:

$$
\mathrm{NSE}=1-\left[\sum_{1}^{N}\left(y_{i}-Y_{i}\right)^{2} / \sum_{1}^{N}\left(y_{i}-Y_{\text {mean }}\right)^{2}\right]
$$

where $y_{\text {mean }}$ represented the mean of the estimated or fitted values of the cross section.

NSE ranges from minus infinity to 1 , where unity NSE indicates the best fit.

\section{Experimental Data of (n, p) and (n, d) Reactions of He-3}

During calculation of the lifetime of neutron [18], protons emitted from the (n, p) reaction of the target He-3 were studied. Also, cross section of this reaction at a range of incident neutron energy $0.15-150 \mathrm{keV}$ was measured [19]. Cross sections for $(\mathrm{n}, \mathrm{p})$ reactions of He-3 by thermal neutrons have been determined in [20], while cross sections for ( $n, p)$ reactions of He-3 by epithermal neutrons have been measured in [21]. Different monoenergetic neutrons between and He3-filled proportional counter were used to obtain the cross sections of both $(n, p)$ and (n, d) reactions of the target He-3 [22]. Indeed, the cross section of (n, d) reaction of He-3 target with $14.4 \mathrm{MeV}$ incident neutron energy has been measured using a counter telescope [23].

\section{Empirical Formulae}

The suggested empirical formulae for (n, p) and (n, d) reactions of He-3 as a function of only incident neutron energy depend on fitting of the observed data by using parametric fitting method. In this method, many different formulae were tried until finding the best parameters of the suggested equation that give 
the best fit of the observed data. Parametric fitting helped us to find parameters for many pre-suggested formulae and only those parameters that gave physical significance were approved. There are no theories to dictate the suggested formulae. Power functions, exponential functions, polynomial function sgaussian functions, and Fourier functions, with different degree, were used to reach the best fit.

\section{Results and Discussion}

Observed cross section data for ${ }^{3} \mathrm{He}(\mathrm{n}, \mathrm{p}){ }^{3} \mathrm{H}$ and ${ }^{3} \mathrm{He}(\mathrm{n}, \mathrm{d}){ }^{2} \mathrm{H}$ described above from the experimental nuclear reaction data EXFOR database of version of 2021 [24]. The best fit for both reactions is shown as:

1) $\mathrm{He}-3(n, d) \mathrm{H}-2$

$$
\sigma(E)=a * e^{b * E}+C * E^{2.1}+d * E^{0.5}+e
$$

where $a=-786, b=-0.6132, c=-0.1607, d=36.7$, and $e=-20.13$.

2) $\mathrm{He}-3(n, p) \mathrm{H}-3$

$$
\sigma(E)=\frac{a}{\sqrt{E}}+b * \sqrt{E}+c
$$

where $a=0.8458, b=1.189, c=-1.174$, and $E \leq 1 \mathrm{MeV}$.

$$
\begin{aligned}
\sigma(E)= & a_{1} e^{\left(\frac{E-b_{1}}{c_{1}}\right)^{2}}+a_{2} e^{\left(\frac{E-b_{2}}{c_{2}}\right)^{2}}+a_{3} e^{\left(\frac{E-b_{3}}{c_{3}}\right)^{2}}+a_{4} e^{\left(\frac{E-b_{4}}{c_{4}}\right)^{2}} \\
& +a_{5} e^{\left(\frac{E-b_{5}}{c_{5}}\right)^{2}}+a_{6} e^{\left(\frac{E-b_{6}}{c_{6}}\right)^{2}}
\end{aligned}
$$

where $a_{1}=0.1342, b_{1}=1.611, c_{1}=0.679, a_{2}=0.05731, b_{2}=2.468, c_{2}=0.7579, a_{3}$ $=0.6842, b_{3}=1.899, c_{3}=3.229, a_{4}=-0.462, b_{4}=2.276, c_{4}=3.161, a_{5}=0.08333, b_{5}$ $=13.5, c_{5}=18.76, a_{6}=1.947, b_{6}=-18.07, c_{6}=16.14$, and $1 \leq E \leq 20 \mathrm{MeV}$.

Equation (13) gives the new empirical cross section $(\mathrm{mb})$ formula as a function of energy $(\mathrm{MeV})$ of the incident neutrons for $(n, d)$, while both Equations (14) and (15) give the new empirical cross section formulae for (n, p) according to two regions of incident neutrons energy. Figure 1 and Figure 2 represent the two reaction formulae graphically coupling with the corresponding observed data.

Also, the discrepancies between the observed data and the calculated were included in Figure 1 and Figure 2.

$$
\Delta(i)=\frac{\sigma_{\text {observed }}\left(E_{i}\right)-\sigma_{\text {calculated }}\left(E_{i}\right)}{\sigma_{\text {observed }}\left(E_{i}\right)}
$$

By applying Equation (9) and Equation (10) to the observed and computed data, the RMSE and NSE values for the three equations are calculated and given in Table 1.

The values of RMSE and NSE of the curve fitting of the cross-sections of the two interactions showed a very high accuracy between the experimental values and the experimental values, given the values of the most important statistical 

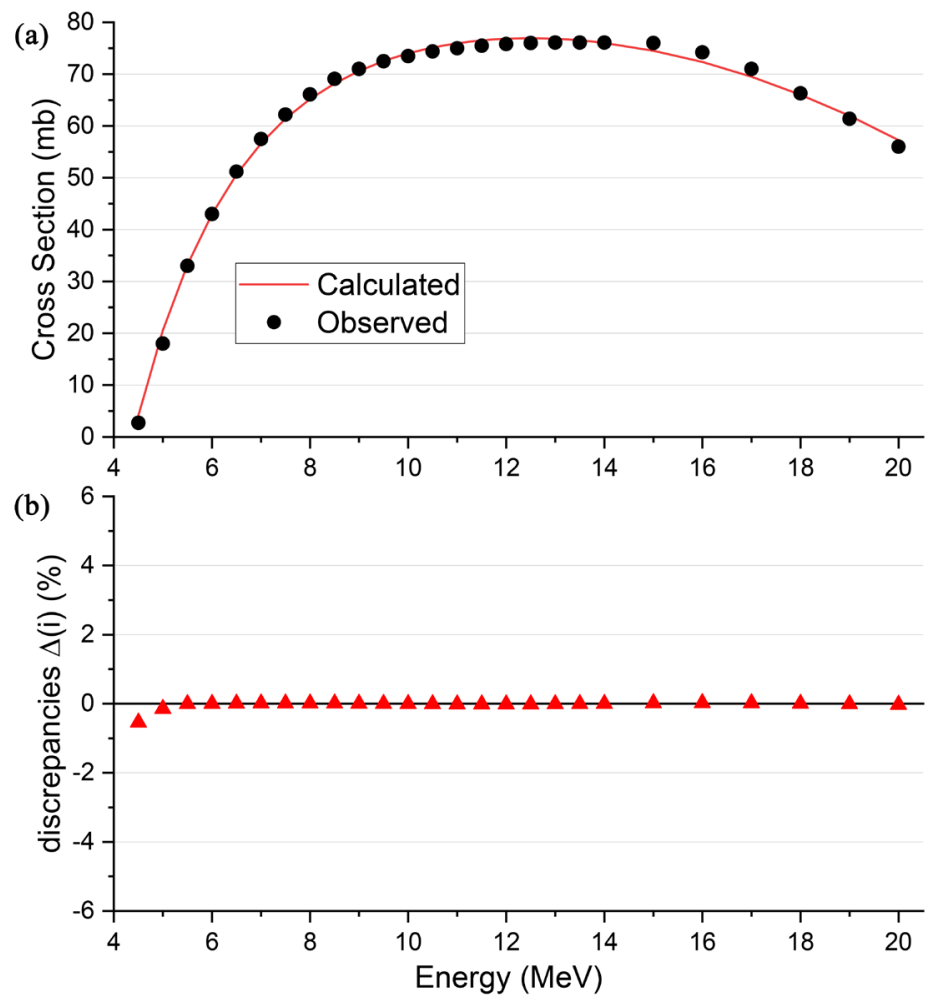

Figure 1. (a) Empirical formula of cross section of ${ }^{3} \mathrm{He}(\mathrm{n}, \mathrm{d}){ }^{2} \mathrm{H}$ Nuclear Reaction as a function of neutron incident energy. (b) Discrepancies between observed and calculated data.
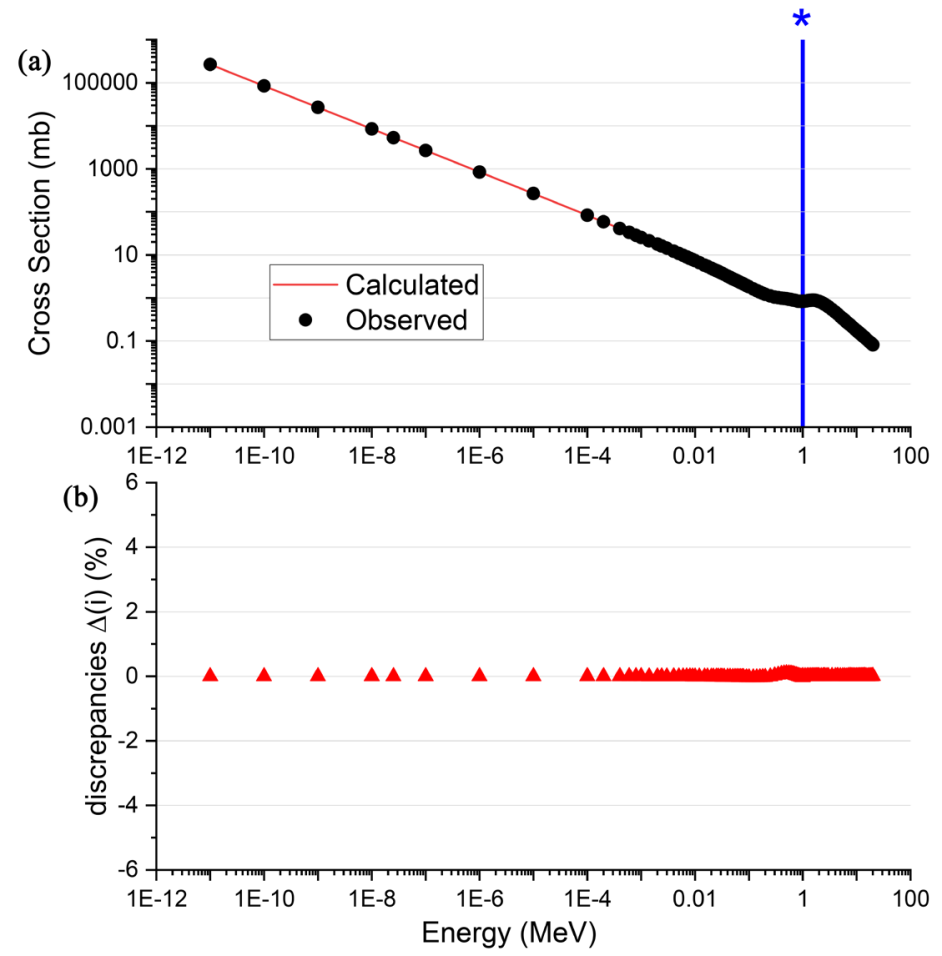

Figure 2. (a) Empirical formula of cross section of ${ }^{3} \mathrm{He}$ (n, p) ${ }^{3} \mathrm{H}$ Nuclear Reaction as a function of neutron incident energy. (b) Discrepancies between observed and calculated data. ${ }^{*}$ Data before the line: $E=1 \mathrm{MeV}$ are taken from Equation (12), while those after the line are taken from Equation (13). 
Table 1. RMSE and NSE values for goodness-of-fit for each case.

\begin{tabular}{ccc}
\hline Nuclear Reaction & \multicolumn{2}{c}{ Goodness of fit indicators } \\
\cline { 2 - 3 } He-3 (n, d) H-2 & $\begin{array}{c}\text { RMSE } \\
\text { Equation (9) }\end{array}$ & $\begin{array}{c}\text { NSE } \\
\text { Equation (10) }\end{array}$ \\
He-3 (n, p) H-3 $(E \leq 1 \mathrm{MeV})$ & 1.16 & 0.9981 \\
He-3 (n, p) H-3 $(1 \leq E \leq 20 \mathrm{MeV})$ & 0.4329 & 1 \\
\hline
\end{tabular}

indicator NSE, which are very close to the integer one in Equation (11) and equal to the integer one in the two Equations (12) and (13). Also, the values of the other indicator RMSE are ideal for Equations (12) and (13) and are considered acceptable for Equation (11).

\section{Summary and Conclusion}

The first step for evaluating the goodness of the new empirical formulae is the visual examination of the fitted curve displayed in couples with the observed data. And it is cleared from both Figure 1 and Figure 2 that the new curves fit the data to a very large extent and that the difference between them is so small which it is difficult to notice. As mentioned before, the closer the value of NSE is to one, the better the result of the curve and it is noted from Table 1 that in all cases its value is either 1 or almost. Also, RMSE indicates to perfect fitting results and the predicted formulae show very good matching with the observed data of He-3 (n, p) H-3 and He-3 (n, d) H-2 reactions.

\section{Conflicts of Interest}

The authors declare no conflicts of interest regarding the publication of this paper.

\section{References}

[1] (1934) Transmutation Effects Observed with Heavy Hydrogen. Proceedings of the Royal Society of London. Series A, Containing Papers of a Mathematical and Physical Character, 144, 692-703. https://doi.org/10.1098/rspa.1934.0077

[2] Kouzes, R.T., Lintereur, A.T. and Siciliano, E.R. (2015) Progress in Alternative Neutron Detection to Address the Helium-3 Shortage. Nuclear Instruments and Methods in Physics Research Section A: Accelerators, Spectrometers, Detectors and Associated Equipment, 784, 172-175. https://doi.org/10.1016/j.nima.2014.10.046

[3] Henzlova, D., Kouzes, R., McElroy, R., Peerani, P., Aspinall, M., Baird, K., Bakel, A., Borella, M., Bourne, M., Bourva, L., Cave, F., Chandra, R., Chernikova, D., Croft, S., Dermody, G., Dougan, A., Ely, J., Fanchini, E., Finocchiaro, P., Zhu, H., et al. (2015) Current Status of Helium-3 Alternative Technologies for Nuclear Safeguards. Office of Scientific and Technical Information (OSTI). https://doi.org/10.2172/1227248

[4] Goncharov, G.A. (2001) The 50th Anniversary of the Beginning of Research in the USSR on the Potential Creation of a Nuclear Fusion Reactor. Physics-Uspekhi, 44, 851-858. https://doi.org/10.1070/PU2001v044n08ABEH001052 
[5] Khvesyuk, V.I., et al. (2001) D-3He Field Reversed Configuration Fusion Power Plant. Fusion Technology, 39, 410-413. https://doi.org/10.13182/FST01-A11963492

[6] Rocha, P.L.F., Correa, S.C.A., Souza, E.M., Silva, A.S., Lima, I.C.B., Lopes, R.T. (2009) Simulation of the $3 \mathrm{He}(\mathrm{n}, \mathrm{p})$ Reaction for Nuclear Well Logging Applications. 11th Congress o Internacional de Geofísica, 24-28 August 2009, 252-255. https://doi.org/10.3997/2214-4609-pdb.195.1889 evt 6year 2009

[7] Fisher, H.O.M. (1989) A Nuclear Cross Section Data Handbook. Office of Scientific and Technical Information (OSTI). https://doi.org/10.2172/5354065

[8] Kodeli, I., Kitsos, S. and Aldama, D.L. (2003) Nuclear Cross Section Library for Oil Well Logging Analysis. International Conference Nuclear Energy for New Europe, Portorož, 8-11 September 2003, 902.1-902.5.

[9] Chadwick, M.B. (1998) Neutron, Proton, and Photonuclear Cross-Sections for Radiation Therapy and Radiation Protection. Radiation and Environmental Biophysics, 37, 235-242. https://doi.org/10.1007/s004110050124

[10] Kannan, U. (2020) Perspectives on Nuclear Data for Advanced Reactor Design and Analysis. Life Cycle Reliability and Safety Engineering, 9, 135-146.

https://doi.org/10.1007/s41872-020-00120-5

[11] Şahan, H., Tel, E. and Yiğit, M. (2014) Neutron Cross Sections Calculations of 7Li Target Nucleus. Journal of Fusion Energy, 34, 16-23. https://doi.org/10.1007/s10894-014-9753-y

[12] Tsvetkov, P. and Waltar, A. (2011) Nuclear Data and Cross Section Processing. In: Fast Spectrum Reactors, Springer, Berlin, 77-109. https://doi.org/10.1007/978-1-4419-9572-8 5

[13] Grossman, M. (1971) Parametric Curve Fitting. The Computer Journal, 14, 169-172. https://doi.org/10.1093/comjnl/14.2.169

[14] Mandal, S. and Uhlmann, V. (2021) A Learning-Based Formulation of Parametric Curve Fitting for Bioimage Analysis. In: Vermolen, F.J. and Vuik, C., Eds., Numerical Mathematics and Advanced Applications ENUMATH 2019, Springer International Publishing, Berlin, 1031-1038. https://doi.org/10.1007/978-3-030-55874-1 102

[15] Nash, J.E. and Sutcliffe, J.V. (1970) River Flow Forecasting through Conceptual Models Part I-A Discussion of Principles. Journal of Hydrology, 10, 282-290. https://doi.org/10.1016/0022-1694(70)90255-6

[16] Zhao, Q., Avdeev, M., Chen, L. and Shi, S. (2021) Machine Learning Prediction of Activation Energy in Cubic Li-Argyrodites with Hierarchically Encoding Crystal Structure-Based (HECS) Descriptors. Science Bulletin. https://doi.org/10.1016/j.scib.2021.04.029

[17] Ritter, A. and Muñoz-Carpena, R. (2013) Performance Evaluation of Hydrological Models: Statistical Significance for Reducing Subjectivity in Goodness-of-Fit Assessments. Journal of Hydrology, 480, 33-45. https://doi.org/10.1016/j.jhydrol.2012.12.004

[18] Kitahara, R., Hirota, K., Ieki, S., Ino, T., Iwashita, Y., Kitaguchi, M., Koga, J., Mishima, K., Morishita, A., Nagakura, N., Oide, H., Otono, H., Seki, Y., Sekiba, D., Shima, T., Shimizu, H.M., Sumi, N., Sumino, H., Taketani, K. and Yoshioka, T. (2019) Improved Accuracy in the Determination of the Thermal Cross Section of $14 \mathrm{~N}(\mathrm{n}, \mathrm{p}) 14 \mathrm{C}$ for Neutron Lifetime Measurement. Progress of Theoretical and EXperimental Physics, 2019, 093C01. https://doi.org/10.1093/ptep/ptz096

[19] Borzakov, S.B., Maletski, K., Pikelner, L.B., Stempinski, M. and Sharapov, E.I. 
(1982) Peculiarities in Deviation from the v-1 Law for $3 \mathrm{He}(\mathrm{n}, \mathrm{p}) \mathrm{T}$ Reaction Cross Section. Excited Level of the 4He Nucleus. Yadernaya Fizika, 35, 532.

[20] Coon, J.H. and Nobles, R.A. (1949) Disintegration of He-3 and N-14 by Thermal Neutrons. Physical Review, 75, 1358-1361. https://doi.org/10.1103/PhysRev.75.1358

[21] Keith, C.D., Chowdhuri, Z., Rich, D.R., Snow, W.M., Bowman, J.D., Penttilä, S.L., Smith, D.A., Leuschner, M.B., Pomeroy, V.R., Jones, G.L. and Sharapov, E.I. (2004) Neutron Cross Sections for $3 \mathrm{He}$ at Epithermal Energies. Physical Review C, 69, Article ID: 034005. https://doi.org/10.1103/PhysRevC.69.034005

[22] Sayres, A.R., Jones, K.W. and Wu, C.S. (1961) Interaction of Neutrons with He-3. Journal of Physical Review, 122, 1853-1863. https://doi.org/10.1103/PhysRev.122.1853

[23] Antolković, B., Paić, G., Tomaš, P. and Rendić, D. (1967) Study of Neutron-Induced Reactions on He-3 at En = 14.4 MeV. Journal of Physical Review, 159, 777-781. https://doi.org/10.1103/PhysRev.159.777

[24] Experimental Nuclear Reaction Data (EXFOR) Database Version of 2021-06-01. https://www-nds.iaea.org/exfor 